\title{
Spata19 Inactivation as a Cause of Oligospermia
}

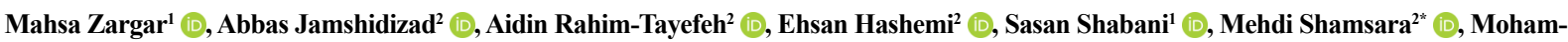 \\ mad Hossein Modarressi ${ }^{* *}$ (i)
}

1. Department of Medical Genetics, School of Medicine, Tehran University of Medical Sciences, Tehran, Iran.

2. Department of Animal Biotechnology, National Insititute of Genetic Engineering and Biotechnology, Tehran, Iran.

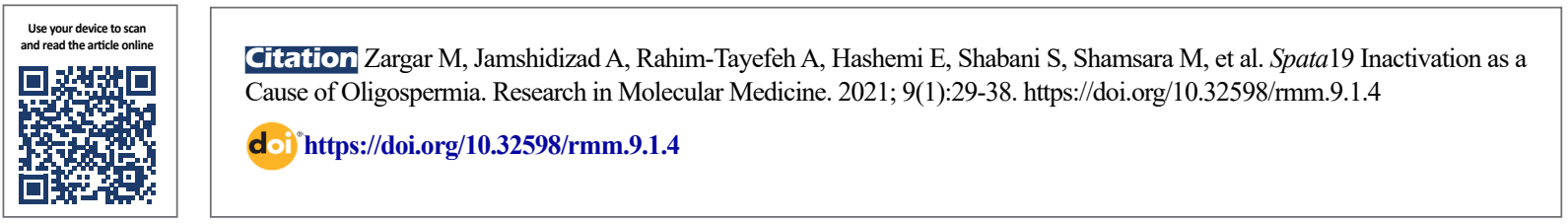

(c) (1) (s)

Article Type:

Research Paper

Article info:

Received: 19 Dec 2020

Revised: 10 Jan 2021

Accepted: 30 Jan 2021

Keywords:

Spata19, CRISPR/Cas9

nickase, Global knockout

mice, Oligospermia, Infertility,

Spermatogenesis, Outbred mice :

\begin{abstract}
A B S T RA C T
Background: Spermatogenesis associated 19 (Spata19) was introduced as a testis-specific gene that was probably involved in spermatogenesis cell apoptosis. Therefore, this study aimed to investigate the effect of Spata19 inactivation on sperm count.

Materials and Methods: We generated global Spata19 knockout mice by CRISPR/Cas9 nickase technology. Disability was validated in three levels of DNA, RNA, and protein using PCR, RT-PCR, and immunohistochemistry. Histological studies were performed for testis. Sperm characteristics were also assessed with CASA software.

Results: Spata 19 knockout mice had a 43 nucleotides deletion in exon 4 of this gene. The presence and absence of Spata 19 were confirmed in normal and knockout mice, respectively. The presence of Spata 19 in normal NMRI mice was detected in the brain, heart, and thymus by semi-nested RT-PCR and in Leydig cells by immunohistochemistry. Histological studies revealed a decrease in sperm count in knockout mice. Also, CASA parameters were significantly reduced $(\mathrm{P}<0.05)$.

Conclusion: These data indicate that Spata 19 inactivation is a cause of oligospermia, and its role could be beyond an adhesive molecule.
\end{abstract}

\section{Introduction}

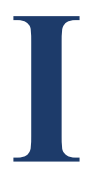

$\mathrm{t}$ is estimated that infertility, as one of the major health problems, affects $15 \%$ of the world's population [1]. Oligospermia is a common finding in men with infertility and refers to semen with low sperm concentrations. Most specimens with low sperm concentrations have significant abnormalities in morphology and motility [2]. Male infertil-

\footnotetext{
* Corresponding Author:

Mehdi Shamsara, PhD.

Address: Department of Animal Biotechnology, National Institute of Genetic Engineering and Biotechnology, Tehran, Iran

Phone: +98 (21) 23872572

E-mail: shamsam2000@gmail
}

ity due to oligozoospermia and azoospermia is associated with some genetic risk factors [3]. Therefore, recognizing the effective genetic factors in fertility can be the basis for diagnosing and treating infertility in the future.

Spata19 is introduced as a testis-specific gene that is probably involved in spermatogenesis, neurogenesis, tumorigenesis, and spermatogenesis cell apoptosis [4-8]. This gene has eight exons and seven introns and is recognized with two transcripts in Mus musculus. These two

Mohammad Hossein Modarressi, PhD.

Address: Department of Medical Genetics, School of Medicine, Tehran University of Medical Sciences, Tehran, Iran.

Phone: +98 (21) 23872572

E-mail: modaresi@tums.ac.ir 


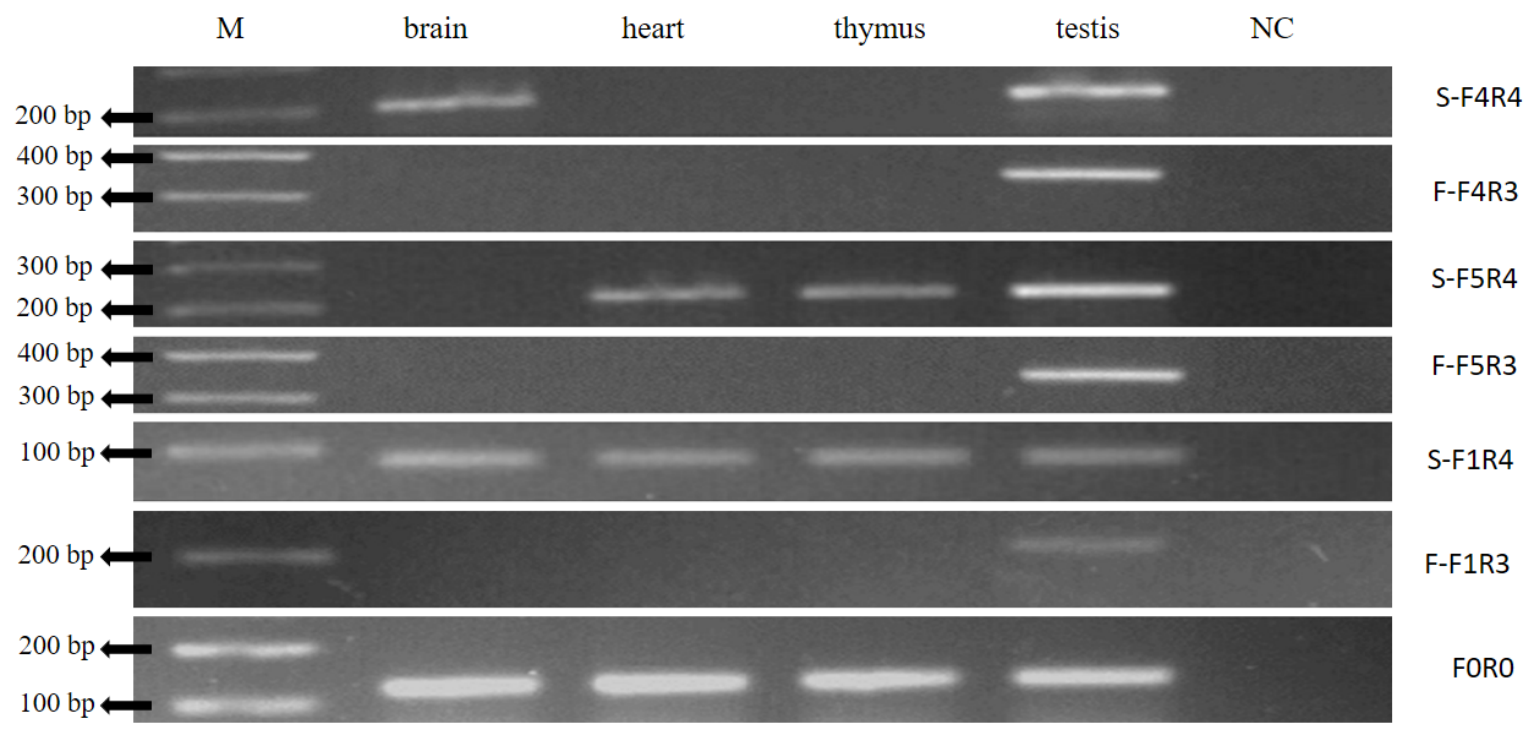

Figure 1. Different transcript variants of Spata19 mRNA in brain, heart, thymus, and testis

Semi-nested RT-PCR was performed in the first (F) and second (S) round with the following primer combination. Then its product was separated on 2\% agarose gel: (F-F1R3 [219bp], S-F1R4 [87bp]) primers amplifying common regions between two transcript variants | (F-F5R3 [352bp], S-F5R4 [220bp]) specific primers for transcript variant 1 (F-F4R3 [342bp], S-F4R4 [210bp]) specific primers for transcript variant 2 | (F0R0 [123bp]) primer pairs for Hprt validating cDNA presence in each lane. Lane M: testis and $\mathrm{NC}$ respectively showed ladder testis sample as positive control and negative control.

transcripts are common in the last five exons but different in the first exon. Transcript 1 (NM_029299.3), whose promoter region has been identified in humans, is expressed in haploid spermatid [9, 10]. Nuclear Factor kappa B $(\mathrm{NF}-\mathrm{\kappa B})$ binding sequence and CAAT box have been determined by comparing human and mouse promoters [11]. Titanium dioxide nanoparticle reduces expression of the gene [12]. Moreover, increased expression of this transcript has been reported in some cancers, including prostate cancer and basal cell carcinoma (BCC) $[5,6]$. The only information about transcript 2 is its increased expression after the meiosis phase of spermatogenesis [7].

These transcripts produce different proteins. Transcript 1 encodes a protein with 154 amino acids containing mitochondrial signal peptide in its N-terminus [13]. This protein is an adhesive molecule that, along with other proteins, is a mitochondrial sheath maintaining factor. Putative localization showed that Spata19 interacts with its counterpart [14]. Transcript 2 (NM_001305058.1) encodes a protein with 119 amino acids (https://www.ncbi. nlm.nih.gov/protein/NP 001291987.1). Functional and structural aspects of both proteins have not been identified.

Knockout mice are useful tools to identify function of unknown genes and to model disease or physiological conditions [15]. Knockout mice can be produced in both global and conditional forms. In conditional knockout mice, a particular gene is inactivated in certain tissues $[16,17]$. This mouse model allows scientists to study diseases that occur in a particular tissue [18]. For example, scientists found the role of Brcal as a tumor suppressor by inactivating the Brcal gene in mice gland tissue [19]. In global knockout mice, a specific gene is deleted in whole mouse cells. Although it has been said that these mice may be excluded from a study due to disruption of their health [19], these mice may be a suitable option for determining the function of testis-specific genes. Because these genes are either not expressed in other tissues or are poorly expressed in other tissues, the risk of deteriorating mouse health is significantly reduced. There are various methods for producing such mice. At top of these methods, CRISPR/Cas was simpler and cheaper to use. Since there was a potential off-target in this method, scientists dramatically reduced that with the double-nicking strategy [20]. In the current study, we investigated effect of Spata19 inactivation on sperm count in NMRI outbred mice which have a close genetic and phenotypic relationship with humans.

\section{Materials and Methods}

\section{Animals}

Adult NMRI mice were purchased from the Pasteur Institute of Iran. The whole process of this study was according to the guidelines for working with laboratory animals 
Table 1. Designed DNA oligonucleotides for genotyping and semi-nested RT-PCR

\begin{tabular}{ccc}
\hline Primer & Sequence (5' $\mathbf{-} \mathbf{3}^{\prime}$ ) \\
\hline F0 & CTCATGGACTGATTATGGACAG \\
\hline F1 & GCAGGTCAGCAAAGAACTTATA \\
F2 & CATGGACATGACATACATGTGACCTGAG \\
F3 & CTCGGAGCATAAGGGAGAAG \\
F4 & GAGGAACAAAGGGGAAGGAAG \\
F5 & ACCGAATTCCCCACCTAGAG \\
R1 & ACCAAGGGTGAACTCGGAC \\
R2 & CATGAGATGAGATACGCTTCGTC \\
R3 & CCAGGCACCATACCTCCAT \\
R4 & CATGAGATGAGATACGCTTCGTC \\
\hline
\end{tabular}

published by the Tehran University of Medical Sciences. The animals were maintained under controlled temperature $\left(25^{\circ} \mathrm{C} \pm 2^{\circ} \mathrm{C}\right)$ with a $12 / 12$ hours light/dark cycle.

\section{Generation of Spata19 knockout mice}

For the generation of Spata19 knockout mice, we used CRISPR/Cas9 nickase genome editing system as previously described [21]. First, using ATUM's gRNA design tool (https://www.atum.bio/eCommerce/cas9/input), two gRNAs and their encoding oligonucleotides were designed for the fourth exon of the genomic sequence, which was the first shared exon between two transcripts. The sense and antisense oligonucleotides of two gRNAs were separately hybridized together (Figure 1), then ligated into the digested pX335 vector (Addgene\#42335) with BbsI. After transforming and cloning the ligated vector in the E. coli bacteria, both plasmids of px335/ gRNA1 and px335 gRNA2 were extracted with EndoFree Plasmid Maxi Kit.

Fertilized eggs were removed from the oviduct of superovulated female mated with wild-type male on the fourth day after vaginal plaque observation. The px335 vectors containing gRNA1 and gRNA2 fragments were mixed with equal concentrations $(2 \mathrm{ng} / \mu \mathrm{L})$ and injected into the male pronucleus of the zygotes, and kept until transfer time in a CO2 incubator. Then, about 35-30 zygotes were transported to the pseudopregnant female oviduct.

\section{Mating and genotyping}

For mating and determining the genotype, 12-16 and 2-3 weeks old mice were used, respectively. The genomic DNA of mice ear and tail biopsies was extracted as previously described [22] and used as templates for F 3 R 2 primers (Table 1) in PCR reaction. To evaluate the fertility of male mice (Spata19+/+, Spata19+/-, Spata19-/-), they were mated with wild-type female mice in a ratio of 1:1. After observing the vaginal plaque, female mice were monitored for fertility for one month.

\section{In vivo fertilization ability of sperm with oocyte}

Male knockout mice with wild-type female mice superovulated by injection of PMSG and HCG were mated. As a positive control, male wild-type mice were simultaneously mated with female wild-type mice on the separate Plexiglas cage. After observing the vaginal plaque, the mice were killed by the cervical dislocation on the same day, and the oocytes were removed from the oviduct. Then 2PN oocytes (containing two pronuclei) were evaluated under the microscope.

\section{Total RNA extraction}

Mice from 8 to 12 weeks were sacrificed by cervical dislocation. The organs of the brain, heart, thymus, and testis were obtained. After homogenization of the organs by freeze and thaw, RNA extraction was carried out with TRIzol (Invitrogen) according to the manufacturer's in- 

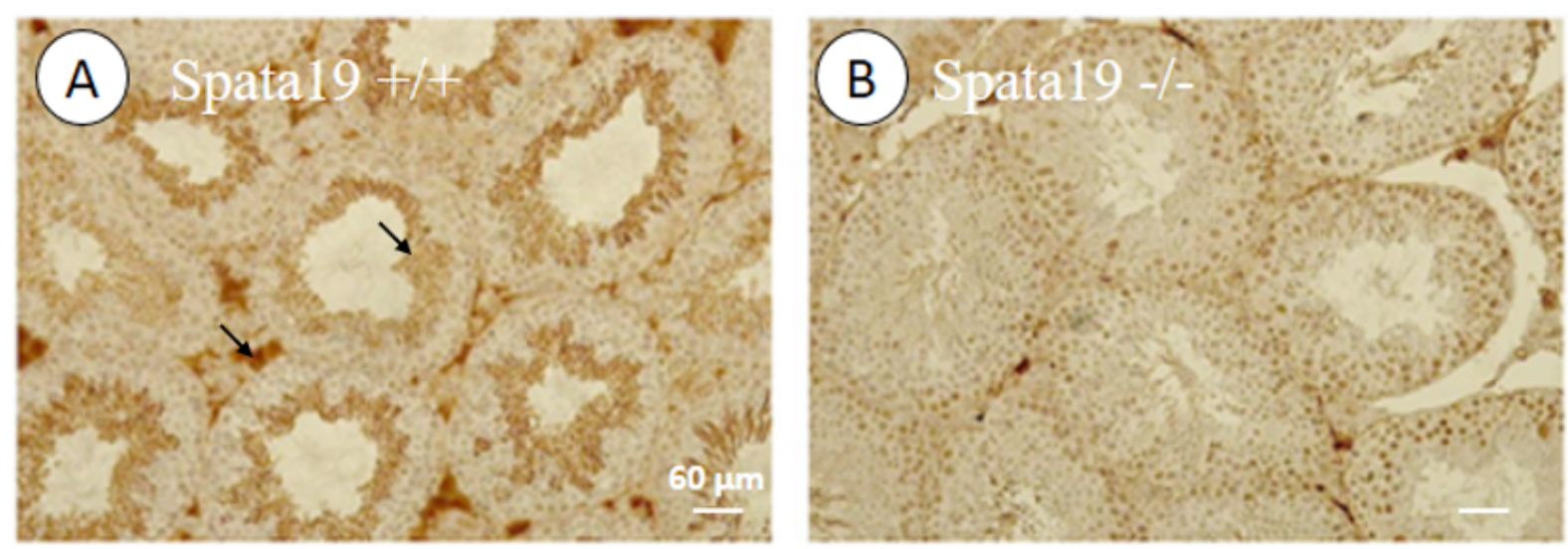

Figure 2. Immunohistochemistry (IHC) staining in Wild-Type (WT) and Spata19-KO testis of mice

A: WT testis showed Spata19 presence in inner cellular layers of seminiferous wall and adjacent structures; B: However that was absent in Spata19-KO testis. Scale bare $=60 \mu \mathrm{m}$.

struction. The concentration and purity of the samples were assessed by NanoDrop ND-2000 (Thermo Fisher Scientific, Wilmington, De), and the quality was evaluated with electrophoresis on 1\% agarose gel (UltraPure TM Agarose; Invitrogen). High-quality RNA samples were stored for up to one week in $-80^{\circ} \mathrm{C}$ for further analysis.

\section{Semi-nested RT-PCR}

Synthesis of single-stranded cDNAs was performed with Yekta Tajhiz Azma cDNA Synthesis Kit ${ }^{\circledR}$, which was contained oligo(dT) and random hexamer primers. About 1500 ng RNA was used for each reaction. To confirm the existence of the synthesized cDNAs, Hprt1 gene amplification with $\mathrm{F} 0 \mathrm{R} 0$ primers was used that this could also demonstrate DNA contamination with resulting in larger bands (3013bp) in comparison with correct $123 \mathrm{bp}$ bands of cDNA (Table 1). The existence of each Spata19's transcript cDNAs was characterized with the help of F1, 5 and 4 R3 primers (Table 1). The amplicons were diluted 1 to 500 and were used as templates for the second round of PCR. In the second PCR, F1, 5 and 4 R4 internal primers were used, respectively (Table 1). The first PCR was 30 cycles and the second PCR was 35 cycles and were carried under the conditions of initial denaturation at $94^{\circ} \mathrm{C}$ for $4 \mathrm{~min} / 30(35)$ cycles of denaturation at $9^{\circ} \mathrm{C}$ for $30 \mathrm{~s}$, annealing at $56^{\circ} \mathrm{C}$ for $30 \mathrm{~s}$ and extension at $72^{\circ} \mathrm{C}$ for $30 \mathrm{~s}$ or final extension at $72^{\circ} \mathrm{C}$ for $5 \mathrm{~min}$. To determine the effect of 43 nucleotides deletion containing the donor splice site (GT), PCR was done for knockout testis using F2R1 primers and was sequenced.
Histological and Immunohistochemistry (IHC) of Testis

Testis of sacrificed mice (Spata19+/+, Spata19+/-) by cervical dislocation method was embedded in paraffin after 48 hours of fixation in Bouin's solution. The sections of $5 \mu \mathrm{m}$ were taken from samples by the microtome. To study the effect of Spata19 knockout on the testis tissue, these sections were stained with hematoxylin and eosin. Then, the samples were studied with a light microscope. To study Spata 19 protein expression, an immunohistochemistry assay was performed in testis tissue. The sections of $5 \mu \mathrm{m}$ were incubated with primary monoclonal antibody Spata19 (Santa Cruz, sc-514325). Then it was treated using the conjugated anti-mouse secondary antibody with horseradish peroxidase (HRP). In the end, staining was done with chromogen 3, 3'-diaminobenzidine (DAB), and the slides were studied under a light microscope.

\section{Sperm Count, Motility, and Morphology}

Cauda epididymis of the killed male mice was removed after opening the abdominal area and was cut by scissors. For swimming up of sperms, the specimens were transferred to an Eppendorf tube containing $1 \mathrm{~mL}$ of TCM medium with $10 \%$ FBS. The microtube containing the sample was placed in a $\mathrm{CO}_{2}$ incubator at $37^{\circ} \mathrm{C}$ for 30 minutes. The sperm samples were washed twice with PBS and centrifuged at $1600 \mathrm{rpm}$ for 10 minutes. Sperm deposition was evaluated by CASA software (Computer-Aided Sperm Analysis) to measure sperm motility parameters. 


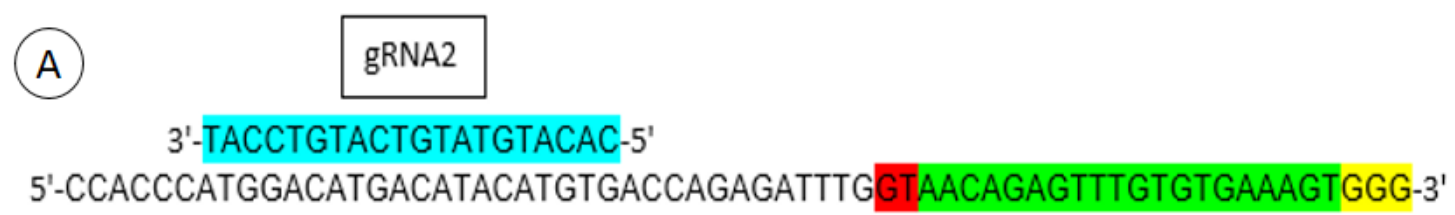

\section{3'-GGTGGGTACCTGTACTGTATGTACACTGGTCTCTAAACCATTGTCTCAAACACACTTTCACCC-5'}

\section{5'-AACAGAGTTTGTGTGAAAGT-3'}

: donor splice site

gRNA1

\section{(B)}

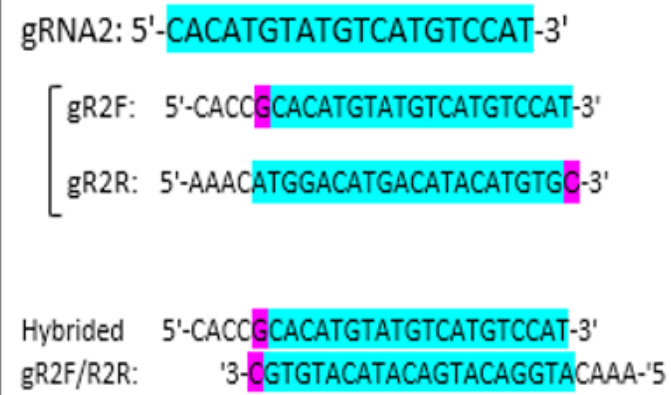

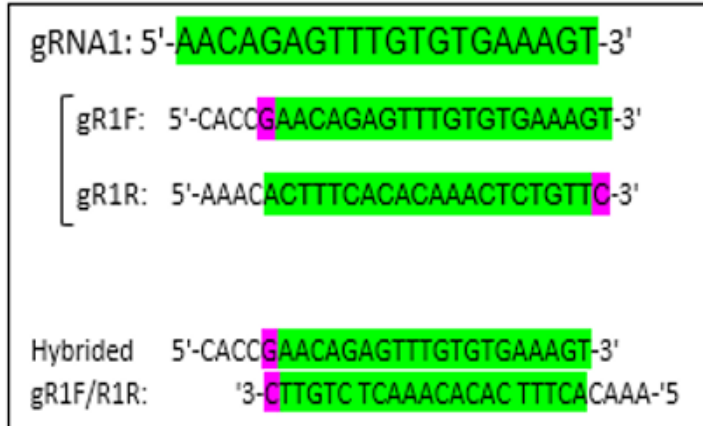

grmm

Figure 3. Designed gRNA Sequence against Spata19 gene and their location on the genome

A: Designed paired gRNAs based on nickase platform were located on the fourth exon and following intron of the genome sequence; B: G nucleotide was added at the 5' end of each gRNA to efficient transcription from the U6 promoter. Also, 5' -CACC and 5' -AAAC sequences were added respectively in 5' ends of each sense and antisense oligonucleotides of each gRNA to ligate paired of them into the digested vector with BbsI.

\section{Modeling and docking}

The 3-D structure of mouse spermatogenesis-associated protein 19 (>NP 083575.1) was simulated using the I-TASSER web server [23]. To simulate interaction between two Spata19 proteins, we focused on our datadriven from docking approach, i.e., HADDOCK using the sequential docking method [24-26]. HADDOCK was an information-driven flexible docking approach for the modeling of biomolecular complexes. It can account for conformational changes occurring upon binding using explicit flexibility during the molecular dynamics' refinement. Since molecular dynamics is not suited for docking purposes because sampling in docking would be extremely computationally expensive, HADDOCK could refine the model [26]. To analyze hydrophobic in- teraction and hydrogen bonds, we used LigPlot [27]. To determine the salt bridge and visualize our model, we used VMDv 1.9.2 [28].

\section{Statistical analysis}

CASA parameters were evaluated by the unpaired $t$ test in GraphPad Prism 8.0.2 software. All data were displayed as Mean \pm SEM. $* \leq 0.05, * * \leq 0.01$ and $* * * \leq 0.001$ were considered as significant $\mathrm{P}$ values. All experiments were repeated at least three times. 


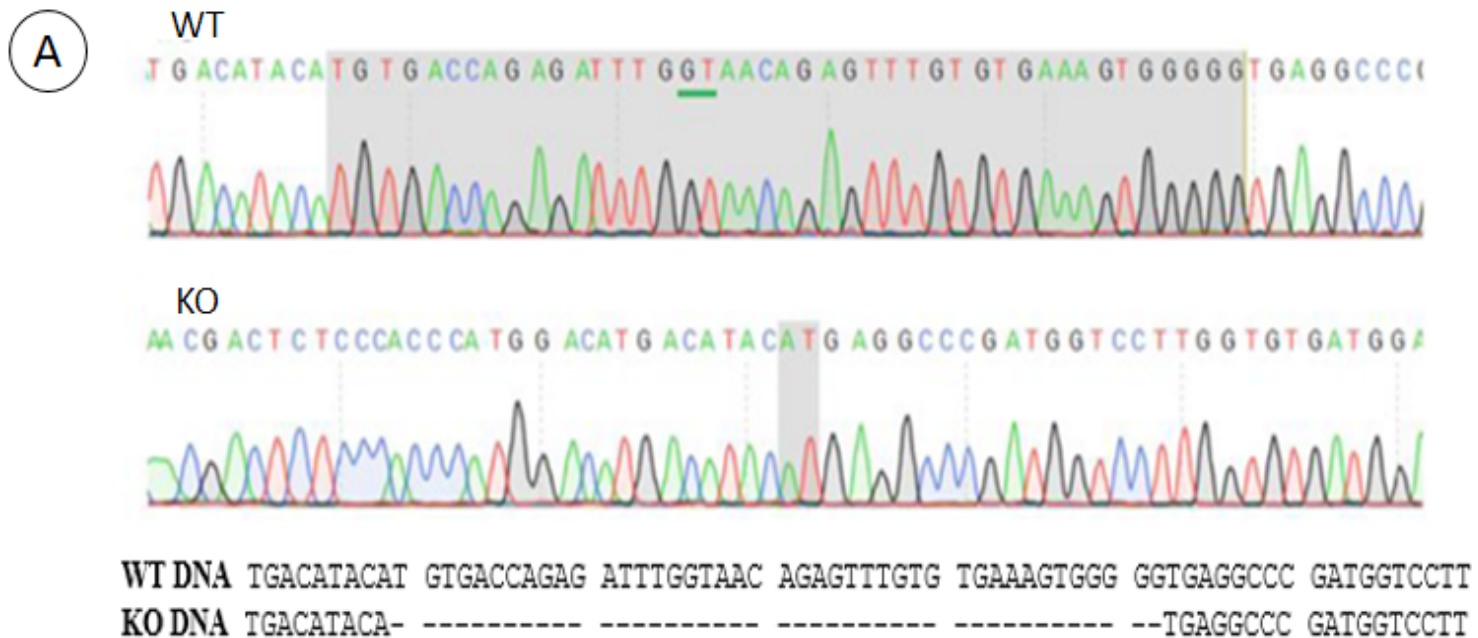

(B) WT DNA atggacatga catacatgtg accagagatt tggtaAcaga gittgtgtga aAgtgggggt

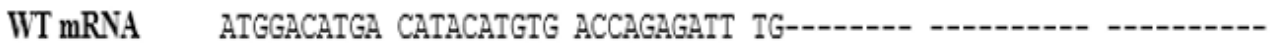

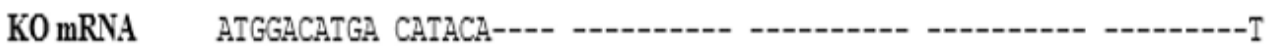

WT DNA GAGGCCCGAT GGTCCTTGGT GTGATGGAC CTGGGCAAAT GGCACATCCC AGCCACAGAT

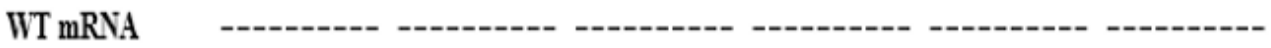

K0 mRNA GAGGCCCGAT GGTCCTTGGT GTGATGGAAC CTGGGCAAAT GGCACATCCC AGCCACAGAT

WT DNA GTCCACATCG GTTAGTAACC ACGTGTATTC CTCTGGATTT CTAGGTGAAA CACCATCTCT

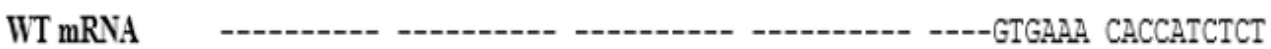

K0 mRNA GTCCACATCG GTTAGTAACC ACGTGTATTC CTCTGGATTT CTAGGTGAAA CACCATCTCT

$\begin{array}{ll}\text { (C) Translation frame of KO } & \text { TEEEASRSIREKMSINDSPTHGHDIHEARTSLVTOP } \\ \text { Translation frame of WT } & \text { TEEEASRSIREKMSINDSPTHGHDIHVTRDLVKHHLSKSDMLTDPSQEVLE } \\ & \end{array}$

Figure 4. Sequence comparison of DNA, mRNA, and protein in wild-type (WT) and Spata19 knockout (KO) mice

A: The genome sequencing results showed 43 bp deletion in a part of the first common exon and its following intron, containing a splice site; B: Those deletions caused part of the intron sequence to remain in mRNA; C: Also, induced premature stop codon prevented the production of both isoforms in $\mathrm{KO}$ mice.

\section{Results}

Spata19 expression in the brain, heart, and thymus in addition to the testis

Spata19 gene expression and transcripts were evaluated in wild-type and homozygous mice by semi-nested RT-PCR. Gene expression in the brain, heart, thymus, and testis was determined with primers designed for common exons. The expression of this gene in the testis was very high, unlike other organs. Each organ has its specific transcripts using primers designed for different regions between the two transcripts (Figure 1).

\section{Spata19 Presence in Leydig Cells}

The presence of Spata19 in Leydig cell was characterized by immunohistochemistry. Compared knockout tissue showed the absence of Spata19 in two regions (Figure 2).

Generation of Global Spata19 Knockout Mice

The CRISPR/Cas9 nickase genome editing system was used to generate global Spata 19 knockout mice by targeting the fourth exon and following intron of the genome sequence, which inactivated the function of all gene's transcripts. Determination of genotype of mice 


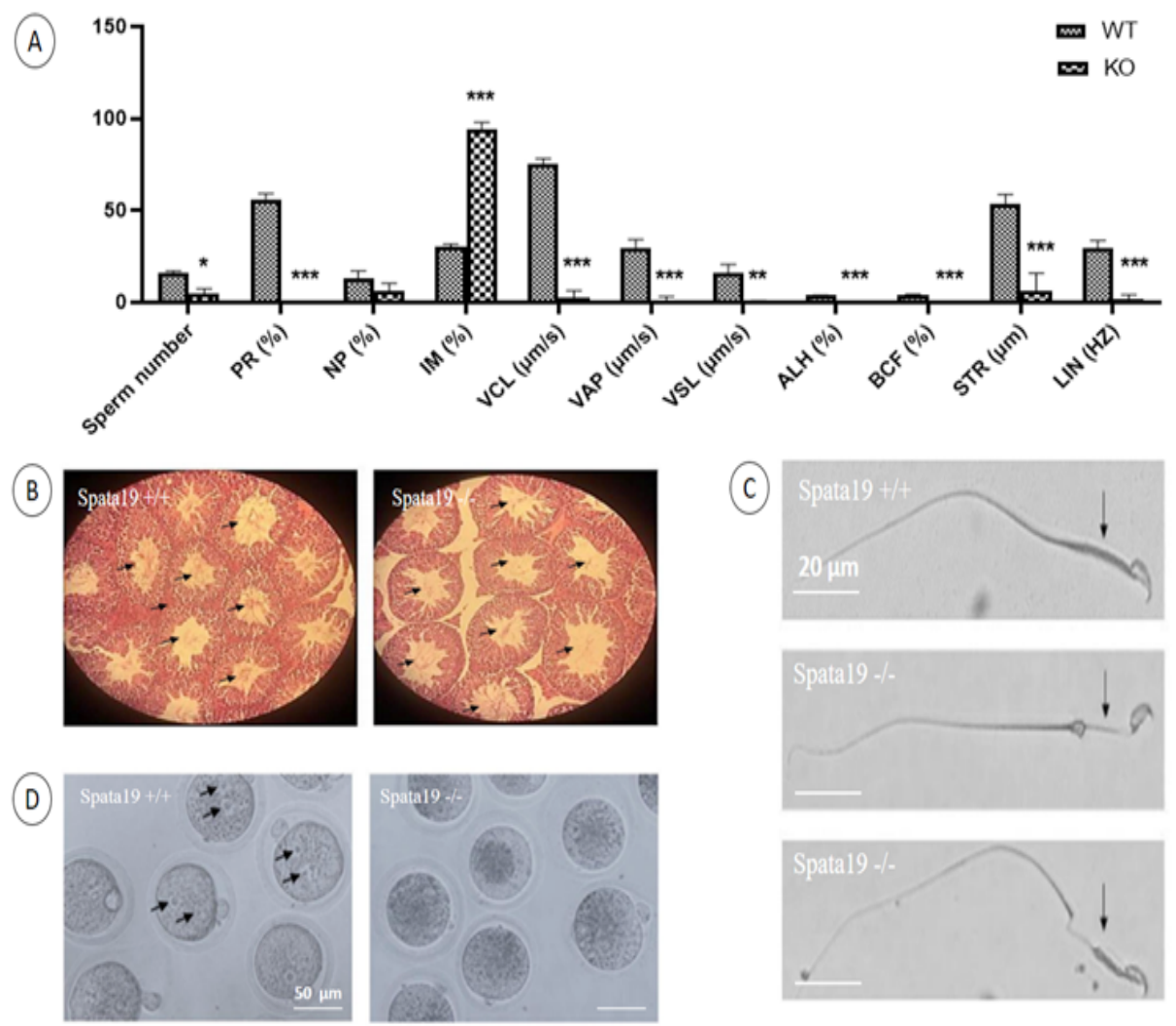

Figure 5. Fertility disorder in Spata19 KO sperm. All evaluation was done using a light microscope

A: CASA parameters include sperm number, the percentage of Progressive Sperm (PR), the percentage of Non-Progressive sperm (NP), the percentage of immotile sperm, Curvilinear Velocity (VCL), Average Path Velocity (VAP), Straight line velocity (VSL), amplitude of lateral head displacement (ALH), Beat-Cross Frequency (BCF), righteousness movement (STR) and linearity coefficient (LIN). Significant P values were shown using ${ }^{*} \leq 0.05,{ }^{* *} \leq 0.01$ and ${ }^{* * *} \leq 0.001$; B: The sections were stained with hematoxylin and eosin. No difference was shown in the seminiferous tube, but significant sperm number reduction was seen in Spata19 knockout mice compared with wild-type mice; C: Abnormality in the midpiece of Spata19 KO sperm Compared to WT sperm, some parts of Spata19 KO-sperm's midpiece had protrusion of outer dense fibers or microtubules. Scale bare=20 $\mu \mathrm{m}$; D: Results of in vivo fertilization showed that Spata19 KO sperms could not fertilize wild-type oocytes. Arrows showed two pronuclei were created as a result of fertilization. Scale bare $=50 \mu \mathrm{m}$.

was performed using 2 primers and PCR. As a result of mating the genotyped male (female) mice (Spata19+/+, Spata19+/-, Spata19-/-) with wild-type female (male) mice, it was found that wild-type and heterozygous mice were fertile, but only the homozygous male mice were infertile. The disruption of Spata19 in knockout mice was confirmed in three levels of DNA, mRNA, and protein using PCR, RT-PCR, and immunohistochemistry (Figure 3, 4). All of these findings indicated the generation of global Spata19 knockout mice.

\section{Oligospermia in Spata19 Knockout Mice}

CASA parameters of sperm from knockout and wildtype mice were evaluated and compared. In this test, the percentage of progressive sperm, the percentage of nonprogressive sperm, the percentage of immotile sperm, curvilinear velocity, average path velocity, straight line velocity, the amplitude of lateral head displacement, beatcross frequency, righteousness movement, and linearity coefficient were compared in wild-type and knockout mice (Figure 5A). Except for non-progressive motility, other parameters were significant. The histologic result of the testis showed that the structure of the knockout mice seminiferous tubules did not significantly differ from the wild-type mice, but sperm count was significantly low (Figure 5B). This result was consistent with the result of sperm count in CASA software. Comparison of the results of the two groups showed a significant reduction in the number of sperms, as well as severe damage to sperm motility indexes in Spata19 knockout mice. 

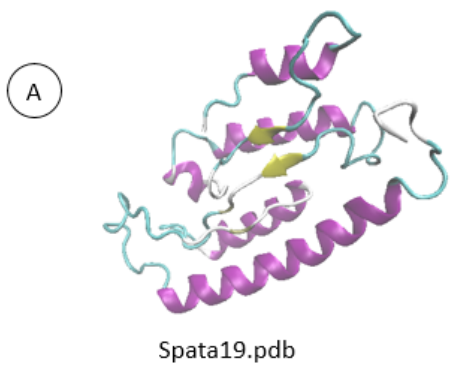

(B)

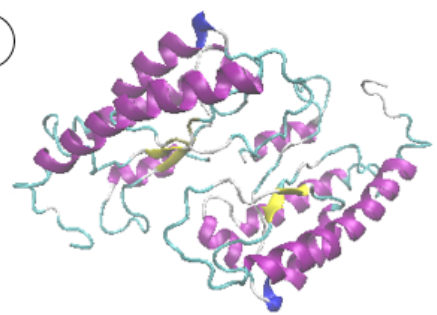

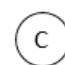

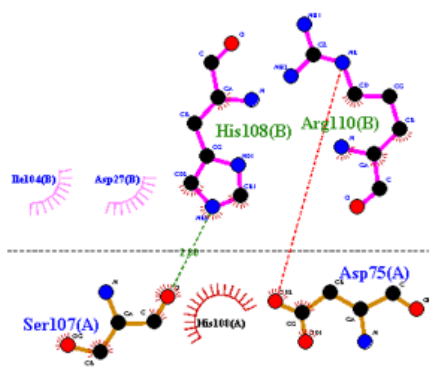

(D)

\begin{tabular}{|l|l|l|}
\hline \multicolumn{2}{|c|}{$\begin{array}{c}\text { Protein-Protein } \\
\text { interaction }\end{array}$} & $\begin{array}{l}\text { Hydrogen } \\
\text { bond }(\AA)\end{array}$ \\
\hline His41 & Thr4 & 2.86 \\
\hline Arg110 & Asn25 & 2.78 \\
\hline Ser107 & His108 & 2.80 \\
\hline
\end{tabular}

Figure 6. Modeling and docking of Spata19 and mGpx4

A: Schematic structure of Spata19 protein modeled with I-TASSER; B: Schematic structure of protein-protein complex which was docked with HADDOCK; C: The binding mode of the protein-protein complex was shown with LigPlot. Hydrogen bond has been shown as green lines; D: All residues involved in the protein-protein interaction using hydrogen bond. Both proteins have been shown using VMD software.

\section{Modeling and docking result}

PDB (Protein Data Bank) structure for Spata19 was predicted with I-TASSER. In this regard, five structures were predicted. The first predicted structure, which had the highest score, was used as the best model (Figure 6A). As described in the Methods and Materials section, the HADDOCK web server was used to predict the interaction between two Spata19 proteins (Figure 6B). LigPlot was used to determine the amino acids involved in hydrogen bonds (Figure 6C, D).

\section{Discussion}

Significant inactivation of some relevant genes such as Spata 19 was associated with spermatogenesis, spermatogenesis cell apoptosis, testicular function, and spermatocyte development revealed by microarray analysis in mouse embryonic stem cells [8]. Because of its high expression in testis, extensive research had been done in this field, but some of its functional and structural aspects were still unknown. In the current study, we found that Spata19 inactivation caused oligospermia or decreased sperm count in global Spata 19 knockout mice generated by CRISPR/Cas9 nickase technology.

In this study, we constructed a global Spata19 knockout mice with CRISPR/Cas9 nickase technology (Figure 3,4). However, germ cell-specific knockout mice generated by Yongjie Mi et al. (2015) with conditional gene-targeting methods would not allow the phenotype examination in other organs [29]. Using Spata 19 knockout mice, we found that only male homozygotes were infertile (as previously reported) and the other females and males were alive and fertile. This suggests that half a dose of Spata 19 was enough for male fertility. To ensure that only Spata19 was inactivated, we examined phenotypes obtained during experiments in other knockout mice with 2 bp deletion [21]. We also used from mutated Cas9 form (D10A or nickase) to prevent probable off-targets. Also, in bioinformatics studies, there was no potential region where gRNAs could be separated at a given distance to create off-target regions. All of these supports the fact that the global knockout Spata19 was created correctly.

Spata 19 had expression in the brain, heart, thymus in addition to the testis (Figure 1). That expression was regulated by two promoters in the first [NC: 2739580027396921] and second [NC: 27396210-27397625] regions based on Genomatix/Gene2Promoter predictions (www.genomatix.de/). So far, only the first region producing transcript 1 was discovered in humans [10]. Comparison of mouse and human Spata 19 genome sequence identified a conserved sequence containing CAAT box and Nuclear Factor kappa B (NF-kB) binding site, which could be important for its specific expression [11]. NF$\kappa \mathrm{B}$ was a transcription factor mainly involved in antiapoptotic response and had a high tendency to interact with titanium dioxide nanoparticles ( $\mathrm{TiO} 2 \mathrm{NPs}$ ) based 
on bioinformatics study [30, 31]. TiO2 NPs-induced reduced Spata 19 expression in testis of mice [12]. Maybe it can be said that NF-KB was a Spata 19 transcription factor that reduced gene expression in the presence of $\mathrm{TiO} 2 \mathrm{NPs}$ due to competitive interaction created between TiO2 NPs and NF- $\mathrm{KB}$ binding sequence to interact with NF- $\mathrm{BB}$ binding site. Therefore, given the expression of NF- $\mathrm{kB}$ in target organs (http://www.ncbi.nlm. nih.gov/UniGene/ESTProfile), that could be considered as a cause of Spata19 gene expression in the brain, heart, thymus, and testis. High expression of the Spata19 gene in the testis suggested its vital role in this organ.

Spata19 was found adjacent to the seminiferous tubules (Figure 2), where Leydig cells producing androgen hormone were located [32]. Our results are similar to the Protein Atlas database (https://www.proteinatlas. org/ENSG00000166118-Spata19/tissue/testis). The previous study has shown that Spata19 inactivation causes schizophrenia-like behavior only in males. One of the leading causes of schizophrenic behavior in males was testosterone, produced by Leydig cells. One of the main elements for testosterone production was ATP. ATP production was decreased in germ cell-specific Spata19 knockout [29, 33].

Spata19 knockout mice had oligospermia (Figure 5A, B). Sperm number was regulated by proliferation and apoptosis during the premeiotic, meiosis, and post-meiotic phases [34]. Spata 19 was introduced as an adhesion molecule that connected adjacent mitochondrial of the sheath using interaction with its counterpart. This protein was one of the mitochondrial sheath maintaining factors. Therefore, in knockout mice, by disrupting this association and altering the mitochondrial physiology, apoptosis may be activated.

\section{Conclusion}

Overall, our study showed that Spata 19 inactivation is a cause of oligospermia. Also, evidence was also found in the presence of Spata19 in locations other than spermatid. These data provided researchers with more information about the pathogenesis and biology of oligospermia.

\section{Ethical Considerations}

\section{Compliance with ethical guidelines}

All experiments were consistent with guidelines for working with laboratory animals published by Tehran University of Medical Science.

\section{Funding}

This study was supported by the Tehran University of Medical Sciences and the National Institute of Genetic Engineering and Biotechnology (Grant No.: 96-04-30-36990).

\section{Authors contribution's}

Conceptualization and supervision: Mehdi Shamsara, Mohammad Hossein Modarressi; Methodology and data collection: All authors; Investigation, writing - original draft, and writing - review \& editing: Mahsa Zargar, Sasan Shabani; Data analysis: Mahsa Zargar; Funding acquisition and resources: Mehdi Shamsara, Mohammad Hossein Modarressi.

\section{Conflict of interest}

The authors declared no conflict of interest.

\section{References}

[1] Ye Y, Wei X, Sha Y, Li N, Yan X, Cheng L, et al. Loss-offunction mutation in TSGA10 causes acephalic spermatozoa phenotype in human.Mol Genet Genomic Med. 2020 8(7):e1284. [DOI:10.1002/mgg3.1284] [PMID] [PMCID]

[2] Daneshmandpour Y, Bahmanpour Z, Hamzeiy H, Moghaddam MM, Moghaddam MM, Khademi B, et al MicroRNAs association with azoospermia, oligospermia, asthenozoospermia, and teratozoospermia: A systematic review. J Assist Reprod Genet. 2020; 37(4):763-75. https:/ / link.springer.com/article/10.1007/s10815-019-01674-9

[3] Dohle GR, Halley DJ, Van Hemel JO, van den Ouwel $\mathrm{AM}$, Pieters $\mathrm{MH}$, Weber RF, et al. Genetic risk factors in infertile men with severe oligozoospermia and azoospermia. Hum Reprod. 2002; 17(1):13-6. [DOI:10.1093/ humrep/17.1.13] [PMID]

[4] McMillan EL, Kamps AL, Lake SS, Svendsen CN, Bhattacharyya A. Gene expression changes in the MAPK pathway in both Fragile $X$ and Down syndrome human neural progenitor cells. Am J Stem Cells. 2012; 1(2):15462. [PMID]

[5] Ghafouri-Fard S, Abbasi A, Moslehi H, Faramarzi N, Taba Taba Vakili S, Mobasheri MB, et al. Elevated expression levels of testis-specific genes TEX101 and Spata19 in basal cell carcinoma and their correlation with clinical and pathological features. Br J Dermatol. 2010; 162(4):772-9. [DOI:10.1111/j.1365-2133.2009.09568.x] [PMID]

[6] Ghafouri-Fard S, Ousati Ashtiani Z, Sabah Golian B, Hasheminasab SM, Modarressi MH. Expression of two testis-specific genes, Spata19 and LEMD1, in prostate cancer. Arch Med Res. 2010; 41(3):195-200. [DOI:10.1016/j. arcmed.2010.04.003] [PMID]

[7] Nourashrafeddin S, Ebrahimzadeh-Vesal R, Modarressi MH, Zekri A, Nouri M. Identification of Spata-19 new variant with expression beyond meiotic phase of mouse 
testis development. Rep Biochem Mol Biol. 2014; 2(2):8993. [PMID] [PMCID]

[8] Nourashrafeddin S, EbrahimzadehVesal R, Miryounesi M, Aarabi M, Zarghami N, Modarressi MH, et al. Analysis of Spata 19 gene expression during male germ cells development, lessons from in vivo and in vitro study. Cell Biol Int Rep. 2013; 21(1): p. 1-7. [DOI:10.1002/cbi3.10010]

[9] Doiguchi M, Mori T, Toshimori K, Shibata Y, Iida H. Spergen-1 might be an adhesive molecule associated with mitochondria in the middle piece of spermatozoa. Dev Biol. 2002; 252(1):127-37. [DOI:10.1006/dbio.2002.0833] [PMID]

[10] Miyamoto T, Sengoku K, Hasuike S, Takuma N, Hayashi $\mathrm{H}$, Yamashita $\mathrm{T}$, et al. Isolation and expression analysis of the human testis-specific gene, SPERGEN-1, a spermatogenic cell-specific gene-1. J Assist Reprod Genet. 2003; 20(2):101-4. [DOI:10.1023/A:1021748310102] [PMID] [PMCID]

[11] Matsuoka Y, Iguchi N, Kitamura K, Nishimura H, Manabe $\mathrm{H}$, Miyagawa $\mathrm{Y}$, et al. Cloning and characterization of a mouse spergen-1 localized in sperm mitochondria. Int J Androl. 2004 27(3):152-60. [DOI:10.1111/j.1365-2605.2004.00466.x] [PMID]

[12] Gao G, Ze Y, Zhao X, Sang X, Zheng L, Ze X, et al. Titanium dioxide nanoparticle-induced testicular damage, spermatogenesis suppression, and gene expression alterations in male mice. J Hazard Mater. 2013; 258-259:133-43. [DOI:10.1016/j. jhazmat.2013.04.046] [PMID]

[13] Doiguchi M, Yamashita H, Ichinose J, Mori T, Shibata Y, Iida H. Complementary DNA cloning and characterization of rat spergen-1, a spermatogenic cell-specific gene-1, containing a mitochondria-targeting signal. Biol Reprod. 2002; 66(5):1462-70. [DOI:10.1095/ biolreprod66.5.1462] [PMID]

[14] Suzuki-Toyota F, Ito C, Toyama Y, Maekawa M, Yao R, Noda $T$, et al. Factors maintaining normal sperm tail structure during epididymal maturation studied in Gopc-/mice. Biol Reprod. 2007; 77(1):71-82. [DOI:10.1095/biolreprod.106.058735] [PMID]

[15] Nazir M, Gaur S, Alsaadi A, Yasser M, Saif M. Knockout Mouse Models for human diseases. Int J Recent SciRes. 2015; 6(7):5517-20. https://www.researchgate.net/profile/ Abdallah-Alsaadi/publication/281522885_KNOCKOUT_ MOUSE_MODELS_FOR_HUMAN_DISEASES/.pdf

[16] Varshney GK, Burgess SM. Mutagenesis and phenotyping resources in zebrafish for studying development and human disease. Brief Funct Genomics. 2014; 13(2):82-94. [DOI:10.1093/bfgp/elt042] [PMID] [PMCID]

[17] Skarnes WC, Rosen B, West AP, Koutsourakis M, Bushell $\mathrm{W}$, Iyer $\mathrm{V}$, et al. A conditional knockout resource for the genome-wide study of mouse gene function. Nature. 2011; 474(7351):337-42. [DOI:10.1038/nature10163] [PMID] [PMCID]

[18] Zhang J, Zhao J, Jiang WJ, Shan XW, Yang XM, Gao JG. Conditional gene manipulation: Cre-ating a new biological era. J Zhejiang Univ Sci B. 2012; 13(7):511-24. [DOI:10.1631/ jzus.B1200042] [PMID] [PMCID]

[19] Clarke AR. Manipulating the germline: Its impact on the study of carcinogenesis. Carcinogenesis. 2000; 21(3):435-41. [DOI:10.1093/carcin/21.3.435] [PMID]

[20] Nasseri S, Nikkho B, Parsa S, Ebadifar A, Soleimani F, Rahimi K, et al. Generation of Fam83h knockout mice by CRIS-
PR/Cas9-mediated gene engineering. J Cell Biochem. 2019; 120(7):11033-43. [DOI:10.1002/jcb.28381] [PMID]

[21] Zargar M, Jamshidizad A, Rahim-Tayefeh A, Hashemi E, Najafi A, Shamsara M, et al. [Generation of global Spata19 knockout mouse using CRISPR/Cas9 nickase technology (Persian)]. Koomesh. 2020; 22(3):380-8. [DOI:10.29252/koomesh.22.3.380]

[22] Zangala T. Isolation of genomic DNA from mouse tails. J Vis Exp. 2007; (6):246. [DOI:10.3791/246] [PMID] [PMCID]

[23] Yang J, Yan R, Roy A, Xu D, Poisson J, Zhang Y. The ITASSER Suite: Protein structure and function prediction. Nat Methods. 2015; 12(1):7-8. [DOI:10.1038/nmeth.3213] [PMID] [PMCID]

[24] Dominguez C, Boelens R, Bonvin AM. HADDOCK: A protein-protein docking approach based on biochemical or biophysical information. J Am Chem Soc. 2003; 125(7):1731-7. [DOI:10.1021/ja026939x] [PMID]

[25] Karaca E, Bonvin AM. A multidomain flexible docking approach to deal with large conformational changes in the modeling of biomolecular complexes. Structure. 2011; 19(4):555-65. [DOI:10.1016/j.str.2011.01.014] [PMID]

[26] de Vries SJ, van Dijk M, Bonvin AM. The HADDOCK web server for data-driven biomolecular docking. Nat Protoc. 2010; 5(5):883-97. [DOI:10.1038/nprot.2010.32] [PMID]

[27] Wallace AC, Laskowski RA, Thornton JM. LIGPLOT: A program to generate schematic diagrams of protein-ligand interactions. Protein Eng. 1995; 8(2):127-34. [DOI:10.1093/ protein/8.2.127] [PMID]

[28] Humphrey W, Dalke A, Schulten K. VMD: Visual molecular dynamics. J Mol Graph. 1996; 14(1):33-8. [DOI:10.1016/02637855(96)00018-5]

[29] Mi Y, Shi Z, Li J. Spata19 is critical for sperm mitochondrial function and male fertility. Mol Reprod Dev. 2015; 82(11):907-13. [DOI:10.1002/mrd.22536] [PMID]

[30] Müller I, Beissert S, Kulms D. Anti-apoptotic NF-kB and "gain of function" mutp53 in concert act pro-apoptotic in response to UVB+IL-1 via enhanced TNF production. J Invest Dermatol. 2015; 135(3):851-60. [DOI:10.1038/jid.2014.481] [PMID] [PMCID]

[31] Ranjan S, Dasgupta N, Sudandiradoss C, Ramalingam C, Kumar A. Titanium dioxide nanoparticle-protein interaction explained by docking approach. Int J Nanomedicine. 2018; 13(T-NANO 2014 Abstracts):47-50. [DOI:10.2147/IJN. S125008] [PMID] [PMCID]

[32] Vasta V, Shimizu-Albergine M, Beavo JA. Modulation of Leydig cell function by cyclic nucleotide phosphodiesterase 8A. Proc Natl Acad Sci U S A. 2006; 103(52):19925-30. [DOI:10.1073/pnas.0609483103] [PMID] [PMCID]

[33] Zargar M, Famitafreshi H, Ara MS, Karimian M, Modarressi MH. Male Spata19 knockout mice have behavioral disorders. Neurol Psychiatry Brain Res. 2020; 38: p. 16-9. [DOI:10.1016/j.npbr.2020.08.007]

[34] Wang H, Zhao R, Guo C, Jiang S, Yang J, Xu Y, et al. Knockout of BRD7 results in impaired spermatogenesis and male infertility. Sci Rep. 2016; 6:21776. [DOI:10.1038/srep21776] [PMID] [PMCID] 